\title{
A CLASS OF ELLIPTIC EQUATIONS WITH INTERIOR DEGENERATION
}

\author{
PATRICK GUIDOTTI
}

\begin{abstract}
A class of linear degenerate elliptic equations inspired by nonlinear diffusions of image processing is considered. It is characterized by an interior degeneration of the diffusion coefficient. It is shown that no particularly natural, unique interpretation of the equation is possible. This phenomenon is reflected in the behavior of numerical schemes for its resolution and points to similar issues that potentially affect its nonlinear counterpart.
\end{abstract}

\section{INTRODUCTION}

The Perona-Malik equation has attracted a fair amount of interest since its introduction in the early 1990s mainly because of an apparent dichotomy between its mathematical ill-posedness and its efficacy as an image processing tool. In the mathematical literature regularizations and relaxations of various kinds have been proposed and analyzed; we refer to [7] for an overview. Here the focus is on the regularization introduced in 8 which replaces the gradient edge detection of Perona-Malik with one using fractional derivatives. The equation reads

$$
\begin{cases}\dot{u}=\nabla \cdot\left(\frac{1}{1+\left|\nabla^{1-\varepsilon} u\right|^{2}} \nabla u\right) & \text { in } B \text { for } t>0, \\ u(0)=u_{0} & \text { in } B,\end{cases}
$$

for a given, fixed $\epsilon \in(0,1]$ and an unknown curve of periodic functions $u(t, \cdot): B \rightarrow \mathbb{R}$ on the normalized unit box (of $\mathbb{R}^{2}$ in applications to image processing). The initial datum $u_{0}$ is a given corrupted image that needs to be enhanced. The classical Perona-Malik equation corresponds to setting $\epsilon=0$ in (1.1) and is known for its strong edge preservation/sharpening capabilities. This is related to its forward-backward nature (see [7] for instance). The distinguishing feature of (1.1) is the combination of its mathematical forward parabolic character, albeit degenerate, and its strong edge preserving properties. Latter are due to the fact that characteristic functions of smooth sets, piecewise constant functions more in general, turn out to be stationary solutions of (1.1). This was the motivation beyond the introduction of (1.1). A transition between non-trivial dynamical behavior for piecewise constant initial data has been observed to occur in numerical experiments as the parameter $\epsilon$ crosses the threshold value $\frac{1}{2}$. If it is smaller, such initial data are preserved, reflecting their equilibrium status for (1.1). If it is larger, however, (numerical) solutions typically (more on this later) tend to exhibit fast convergence to a uniform state. In the context of smooth solutions, this transition from local to global well-posendess was analytically confirmed in [6], where global existence and convergence to trivial steady-states are established for smooth enough initial data for a variant of (1.1) in a one-dimensional setting. This is somewhat unsatisfactory since most interesting (numerical) solutions of (1.1) are not smooth enough and, while piecewise constant solutions can be viewed as stationary for the evolution, no weak solvability theory is available for any low regularity class of functions including them. A significant impediment to the development of a comprehensive weak solvability theory is the (conjectured) non-existence of function spaces containing piecewise constant functions for which weak solutions can be constructed. In this paper,

1991 Mathematics Subject Classification. 35J20, 35J25, 35J70, 35K20, 35 K65.

Key words and phrases. Weakly degenerate, elliptic, analytic semigroups, regularization. 
the focus is on a class of linear equations closely related to a modification of (1.1) given by

$$
\begin{cases}\dot{u}=\nabla \cdot\left(\frac{1}{1+N_{\varepsilon}^{2}(|\nabla u|)} \nabla u\right)=\nabla \cdot\left(a_{\varepsilon}(u) \nabla u\right) & \text { in } B \text { for } t>0, \\ u(0)=u_{0} & \text { in } B,\end{cases}
$$

where the convolution operator $N_{\varepsilon}$ is a Fourier multiplication operator defined through

$$
N_{\varepsilon}=\mathcal{F}^{-1} \operatorname{diag}\left[\left(|k|^{-\varepsilon}\right)_{k \in \mathbb{Z}^{n} \backslash\{0\}}\right] \mathcal{F} \sim \frac{1}{|x|^{n-\varepsilon}} *,
$$

for $n=1,2$, where $\mathcal{F}$ denotes the Fourier transform on $\mathrm{L}_{\pi}^{2}(B)$. As a step towards understanding this nonlinear equation for relevant non-smooth initial data, one can consider an initial datum in the form of a characteristic function of a subset of the circle $(n=1)$ or of the torus $(n=2)$, with smooth boundary $\Gamma$, and study the linear equation

$$
\begin{cases}\dot{u}=\nabla \cdot\left(a_{\varepsilon}\left(u_{0}\right) \nabla u\right) & \text { in } B \text { for } t>0, \\ u(0)=u_{0} & \text { in } B\end{cases}
$$

It will be shown that, in this case,

$$
N_{\varepsilon}(|\nabla u|)(x) \sim \frac{1}{d(x, \Gamma)^{1-\varepsilon}} \text { as } x \sim \Gamma,
$$

for the distance $d(x, \Gamma)$ to the boundary $\Gamma$ and thus that

$$
a_{\varepsilon}\left(u_{0}\right)=\frac{1}{1+N_{\varepsilon}^{2}\left(\left|\nabla u_{0}\right|\right)} \sim d(x, \Gamma)^{2-2 \varepsilon} \text { as } x \sim \Gamma,
$$

would hold for the corresponding diffusivity. It will be shown that, for $\varepsilon>\frac{1}{2}$, equation (1.4) possesses a unique solution which instantaneously regularizes and eventually converges to a trivial steady-state. Since certain piecewise constant functions can also be seen as steady-states, nonuniqueness ensues. While it seems natural to view (1.4) as "the" gradient flow engendered by the energy functional given by

$$
\int_{B} \alpha|\nabla u|^{2} d x,
$$

for $\alpha=a_{\varepsilon}\left(u_{0}\right)$ and $\varepsilon>1 / 2$, latter does not appear to have a preferred, unique domain of definition. For this reason, it cannot be claimed that (1.4) possesses a natural and unambiguous interpretation. It is in fact possible to construct three distinct gradient flows compatible with the above energy which exhibit different behaviors. One which regularizes initial data immediately and averages them out exponentially fast, as is naturally expected of a heat equation, and, others, which preserves certain discontinuities forever. This ambiguity is reflected at the numerical level as a grid-choice phenomenon. In other words different solutions can be observed numerically even with the same type of discretization depending on the choice of discretization points. In the "regularizing interpretation", the degenerate elliptic operator can be shown to generate an analytic semigroup on $\mathrm{L}_{\pi}^{2}$. In this case, the evolution can be viewed as the vanishing viscosity limit for the equation with diffusivity $\delta+a_{\varepsilon}\left(u_{0}\right)$ for $\delta>0$. While this is possibly the most natural interpretation of the degenerate equation, others cannot be neglected as they could help explain numerical observations. In fact, many nonlinear diffusions have been utilized in image processing especially because of their ability to preserve edges. This paper shows that, even in the linear case, extreme care is required when using such methods as they are not assured to deliver consistent results, nor do they provide assurances that the output images possess properties that are naturally connected to the underlying "true" image that one is purportedly trying to recover. This will be demonstrated with a simple one dimensional discretization. 
Elliptic and parabolic equations with interior degenerations have not been studied extensively in the literature. The approach taken in this paper is most akin to that utilized by [3] in a onedimensional context in that it shows, in particular, generation of an analytic semigroup on $\mathrm{L}^{2}$. Recently, a general framework for linear and nonlinear degenerate parabolic equations has been developed in [1] using different techniques based on the concept of singular manifolds. These techniques can be adapted to construct one of the possible solutions in a nonlinear context. This is done in 9 .

The paper is organized as follows. In the next two sections, it is shown how the seemingly natural energy functional for (1.4) admits distinct and valid interpretations which lead to different evolutions. In the regularizing case, the associated operator will be shown to generate analytic, contraction semigroups. Spectral properties related to compact embeddings and the validity of a Poincaré inequality will be highlighted. Additionally, two different flows will be presented which can preserve singularities. In Section 4 the one-dimensional case will be considered to show how numerical implementations can indeed produce at least two distinct types of solutions. Interestingly, one of them is incompatible with any of the interpretations presented in Section 3. It turns out to be compatible with strongly degenerate equations. Section 5 deals with the vanishing viscosity limit via $\Gamma$-convergence.

\section{The Setup and The Energy Functional}

The main focus of this paper is on linear weakly degenerate elliptic problems with diffusivity belonging to a specific class of functions. Let $n=1,2$ and $B=[-1,1)^{n}$ be the periodicity box. Consider bounded periodic functions $\alpha: B \rightarrow[0, \infty)$ which vanish only on a smooth closed curve $\Gamma \subset B$, if $n=2$, or on $\Gamma=\{ \pm 1 / 2\}$, if $n=1$, and satisfy

$$
\frac{1}{c} d(x, \Gamma)^{\sigma} \leq \alpha(x) \leq c d(x, \Gamma)^{\sigma}, x \in B,
$$

for some $1 \leq c<\infty, \sigma \in[0,1$ ), and that are otherwise smooth on $B \backslash \Gamma$ (at least Hölder continuous of exponent $\sigma$, if not stated otherwise). The function $d(\cdot, \Gamma)$ given by

$$
d(x, \Gamma)=\inf _{y \in \Gamma}|x-y|, x \in B
$$

represents the distance function to the set $\Gamma$. The collection of all coefficient functions $\alpha$ of the above type is denoted by $\mathrm{D}_{\pi}^{\sigma}$. For $\alpha \in \mathrm{D}_{\pi}^{\sigma}$ consider the elliptic problem

$$
\left\{\begin{array}{l}
-\nabla \cdot(\alpha(x) \nabla u)=f \text { in } B, \\
u \text { periodic }
\end{array}\right.
$$

for $f \in \mathrm{L}_{\pi}^{2}(B)$, the space of functions which are square integrable and periodic (hence the subscript $\pi)$. The corresponding evolutionary problem, given by

$$
\left\{\begin{array}{l}
\dot{u}-\nabla \cdot(\alpha(x) \nabla u)=0 \quad \text { in } B, \\
u \text { periodic }
\end{array}\right.
$$

is also of interest. In the case that $\sigma=0$, the diffusivity cannot obviously be required to vanish on $\Gamma$ and equation (2.2) is strongly elliptic, while, for $\sigma \in(0,1)$, it is a so-called weakly degenerate elliptic problem. For this nomenclature and basic results in the elliptic case, it is refered to [10, where a weakly degenerate equation of type (2.2) is characterized in particular by the conditions that

$$
0 \leq \alpha \in \mathrm{L}^{1}(B) \text { and that } \frac{1}{\alpha} \in \mathrm{L}^{1}(B) .
$$


Problems (2.2) and (2.3) are closely related to the energy functional

$$
E_{\alpha}(u)=\int_{B} \alpha|\nabla u|^{2} d x
$$

Observe that this functional can be thought of as being defined on the weighted space

$$
\mathrm{H}_{\pi, \alpha}^{1}(B):=\left\{u \in \mathrm{L}_{\pi}^{2}(B):|\nabla u| \in \mathrm{L}_{\pi}^{1}(B) \text { and } \int \alpha(x)|\nabla u(x)|^{2} d x<\infty\right\}
$$

which is a Banach space with respect to the norm

$$
\|u\|_{\mathrm{H}_{\pi, \alpha}^{1}(B)}:=\left(\|u\|_{2}^{2}+\|\sqrt{\alpha} \nabla u\|_{2}^{2}\right)^{1 / 2}
$$

since the requirement that $\nabla u$ be a regular distribution does not need to be reiterated in the norm in view of the validity of

$$
\int_{B}|\nabla u| d x \leq\left(\int_{B} \frac{1}{\alpha(x)} d x\right)^{1 / 2}\left(\int_{B} \alpha|\nabla u|^{2} d x\right)^{1 / 2}, u \in \mathrm{H}_{\pi, \alpha}^{1}(B),
$$

thanks to the Cauchy-Schwarz inequality. On the other hand, if the requirement that $|\nabla u|$ be integrable is dropped, the energy functional can be viewed as being defined on

$$
\widetilde{\mathrm{H}}_{\pi, \alpha}^{1}(B):=\left\{u \in \mathrm{L}_{\pi}^{2}(B): \nabla u \in \mathcal{M}_{\pi}(B) \text { and } \sqrt{\alpha}|\nabla u| \in \mathrm{L}_{\pi}^{2}(B)\right\},
$$

where $\mathcal{M}_{\pi}(B)$ is the space of periodic (vector-valued) Radon measures on $B$, dual to the space $\mathrm{C}_{\pi}(B)^{n}$ of periodic continuous functions on $B$, or, even on the larger space obtained simply requiring that $\sqrt{\alpha} \nabla u$ be square integrable for the distributional gradient of $u$. The main reason to consider the space $\widetilde{\mathrm{H}}_{\pi}^{1}(B)$ along with the functional $E_{\alpha}$, which will be denoted by $\widetilde{E}_{\alpha}$ if considered with this domain, is that it contains the characteristic function $\chi_{\Omega}$ of the smooth domain $\Omega$ bounded by the curve $\Gamma$ for $n=2$ or, of the interval $[-1 / 2,1 / 2]$ for $n=1$. Indeed, one has that

$$
\nabla \chi_{\Omega}=\nu_{\Gamma} \delta_{\Gamma} \text { and } \chi_{\Omega}^{\prime}=\delta_{1 / 2}-\delta_{-1 / 2}
$$

for $n=2$ and $n=1$, respectively, and therefore that $\sqrt{\alpha} \nabla \chi_{\Omega}=0$ as well as $\sqrt{\alpha} \chi_{[-1 / 2,1 / 2]}^{\prime}=0$. Here $\nu_{\Gamma}$ denotes the unit outward normal to $\Gamma$, while $\delta_{\Gamma}$ represents the line integral distribution along $\Gamma$. Observe that these functions are non-trivial minimizers of $\widetilde{E}_{\alpha}$ and they might play a role in the evolution of the corresponding gradient flow. Equation (2.2) could arguably also be interpreted as a system for a pair $\left(u_{i}, u_{o}\right)$ of functions defined on the connected components $\Omega_{i}=\Omega$ and $\Omega_{o}$ of $B \backslash \Gamma$ and belonging to the space

$$
\overline{\mathrm{H}}_{\pi, \alpha}^{1}(B):=\mathrm{H}_{\alpha}^{1}\left(\Omega_{i}\right) \times \mathrm{H}_{\pi, \alpha}^{1}\left(\Omega_{O}\right),
$$

and where the energy functional is now interpreted as

$$
\bar{E}_{\alpha}\left(u_{i}, u_{0}\right)=\int_{\Omega_{i}} \alpha\left|\nabla u_{i}\right|^{2} d x+\int_{\Omega_{0}} \alpha\left|\nabla u_{o}\right|^{2} d x=E_{\alpha}\left(u_{i}\right)+E_{\alpha}\left(u_{o}\right),
$$

where the last identity holds with the understanding that the energy functionals are for functions with the appropriate domain of definition. This last interpretation is justified by the fact that $\mathrm{L}_{\pi}^{2}(B)=\mathrm{L}^{2}\left(\Omega_{i}\right) \oplus \mathrm{L}_{\pi}^{2}\left(\Omega_{o}\right)$, so that the energy functional, if extended by the value $\infty$, can be thought of as being defined on $\mathrm{L}_{\pi}^{2}(B)$. 


\section{The Different Flows}

3.1. The Regularizing Case. It is easily seen that that compactly supported test functions belong to $\mathrm{H}_{\pi, \alpha}^{1}(B)$, i.e. that

and that

$$
\mathcal{D}(B) \subset \mathrm{H}_{\pi, \alpha}^{1}(B),
$$

$$
\mathcal{D}_{\pi}(B)=\mathrm{C}_{\pi}^{\infty}(B) \subset \mathrm{H}_{\pi, \alpha}^{1}(B),
$$

where the subscript $\pi$ in the first space indicates that periodic test-functions are considered. It is natural to view (2.2) with $f \equiv 0$ as the stationarity condition for $E_{\alpha}$ given by (2.4) and defined on $\mathrm{H}_{\pi, \alpha}^{1}(B)$. Latter happens to be the natural space which makes the functional coercive (see below). The form associated to $E_{\alpha}$ is given by

$$
a_{\alpha}(u, v):=\int_{B} \alpha \nabla u \cdot \nabla v d x, u, v \in \mathrm{H}_{\pi, \alpha}^{1}(B),
$$

and induces the operator

$$
\mathcal{A}_{\alpha}: \mathrm{H}_{\pi, \alpha}^{1}(B) \rightarrow \mathrm{H}_{\pi, \alpha}^{1}(B)^{\prime}=: \mathrm{H}_{\pi, \alpha}^{-1}(B),
$$

given by

$$
\mathcal{A}_{\alpha} u:=\left[v \mapsto \int_{B} \alpha \nabla u \cdot \nabla v d x\right] \in \mathrm{H}_{\pi, \alpha}^{-1}(B) .
$$

Clearly the form $a_{\alpha}$ is non-negative and symmetric. Next a few properties of the space $\mathrm{H}_{\pi, \alpha}^{1}(B)$ are collected which are important for the understanding of the weakly degenerate problem (2.2). Notice that proofs are mostly given for $n=2$ since the one dimensional case is simpler and can be handled in a perfectly analogous manner.

Take a compactly supported, radial and radially decreasing, non-negative, smooth testfunction $\varphi \in \mathcal{D}\left(\mathbb{R}^{n}\right)$ with $\operatorname{supp}(\varphi) \subset B$ and with

$$
\int_{B} \varphi(y) d y=1
$$

Define an associated mollifier $\varphi_{m}$ in the usual way

$$
\varphi_{m}(x)=m \varphi(m x) \text { and } u_{m}(x)=\varphi_{m} *_{\pi} u(x):=\int_{B} \varphi_{m}(x-y) u(y) d y, x \in B .
$$

Alternatively, one can think of the convolution on the torus and write

$$
\int_{B} \varphi_{m}(x-y \mid 2) u(y) d y, x \in B,
$$

where $(x-y \mid 2)$ denotes addition modulo 2 component by component. In order not to overburden the notation, the subscript $\pi$ in the convolution will be dropped.

Lemma 3.1. It holds that $\alpha \varphi_{m} * \frac{1}{\alpha} \in \mathrm{L}_{\pi}^{\infty}(B)$

Proof. Consider first the one dimensional case $n=1$. Fix $\delta>0$ such that

$$
\frac{1}{c}|x \pm 1 / 2|^{\sigma} \leq \alpha(x) \leq c|x \pm 1 / 2|^{\sigma},
$$

for $c \geq 1$ and $|x \pm 1 / 2| \leq 3 \delta$. Now, if $|x \pm 1 / 2| \geq 2 \delta$, one has that

$$
\alpha(x) \varphi_{m} * \frac{1}{\alpha} \leq \frac{c}{2^{\sigma} \delta^{\sigma}}\|\alpha\|_{\infty},
$$

provided $m \geq 1 / \delta$. If, on the other hand $|x-1 / 2|<2 \delta$ (the case when $|x+1 / 2|<2 \delta$ can be handled in the same way), then

$$
x \in[1 / 2-2 / m, 1 / 2+2 / m] \cup \bigcup_{k \geq 2}[1 / 2-(k+1) / m, 1 / 2-k / m] \cup[1 / 2+k / m, 1 / 2+(k+1) / m],
$$


where the union ends when the interval $[1 / 2-2 \delta, 1 / 2+2 \delta]$ is completely covered. While a finite number of intervals suffice for any finite $m$, the number increases with $m$. If $x$ belongs to the interval $[1 / 2-2 / m, 1 / 2+2 / m]$, one has that

$$
\begin{aligned}
\alpha(x) \int_{x-1 / m}^{x+1 / m} \frac{\varphi_{m}(x-y)}{\alpha(y)} d y & \leq m \int_{x-1 / m}^{x+1 / m} \frac{|x-1 / 2|^{\sigma}}{|y-1 / 2|^{\sigma}} d y \\
& \leq c m^{1-\sigma}\left[(1 / 2-x+1 / m)^{1-\sigma}+(x+1 / m-1 / 2)^{1-\sigma}\right] \leq c<\infty .
\end{aligned}
$$

If, on the other hand, $x \in[1 / 2+k / m, 1 / 2,+(k+1) / m]$ (or similarly, if $x$ belongs to the interval $[1 / 2-(k+1) / m, 1 / 2-k / m])$, it holds that

$$
\begin{aligned}
\alpha(x) \int_{x-1 / m}^{x+1 / m} \frac{\varphi_{m}(x-y)}{\alpha(y)} d y & \leq m \int_{x-1 / m}^{x+1 / m} \frac{\alpha(x)}{\alpha(y)} d y \leq c m|x-1 / 2|^{\sigma} \int_{x-1 / m}^{x+1 / m} \frac{1}{|y-1 / 2|^{\sigma}} d y \\
& \leq c\left(\frac{k+2}{m}\right)^{\sigma}\left(\frac{m}{k-1}\right)^{\sigma} \leq c\left(\frac{k+2}{k-1}\right)^{\sigma} \leq c<\infty, k \geq 2 .
\end{aligned}
$$

Since there is no restriction on $k$, the estimate is valid for any (large) $m$ and the proof is complete for $n=1$.

As for $n=2$, since $\Gamma$ is assumed to be a smooth, closed curve, it possesses a tubular neighborhood $T_{\Lambda}(\Gamma)$ with coordinates $(y, \lambda)$ determined by

$$
T_{\Lambda}(\Gamma)=\left\{y+\lambda \nu_{\Gamma}(y) \mid y \in \Gamma, \lambda \in(-\Lambda, \Lambda)\right\},
$$

where $\nu_{\Lambda}$ is the unit outward normal to $\Gamma$. Then, for any $x \in T_{\Lambda}(\Gamma)$, it is possible to find a unique pair $(y(x), \lambda(x)) \in \Gamma \times(-\Lambda, \Lambda)$ such that

$$
x=y(x)+\lambda(x) \nu_{\Gamma}(y(x)) .
$$

It follows that any integral with respect to the two-dimensional Lebesgue measure $d x d y$ amounts to an integral in the new coordinates with respect to the measure $d \sigma_{\Gamma_{\lambda}}(y) d \lambda$, where $\sigma_{\Gamma_{\lambda}}$ is the line measure along

for $\lambda \in(-\Lambda, \Lambda)$. Notice that

$$
\Gamma_{\lambda}=\left\{y+\lambda \nu_{\Gamma}(y) \mid y \in \Gamma\right\}
$$

$$
d \sigma_{\Gamma_{\lambda}}=\left|\dot{\gamma}_{\lambda}(t)\right| d t
$$

for any parametrization $\gamma_{\lambda}$ of $\Gamma_{\lambda}$. Denote by $\gamma$ the arc-length parametrization of $\Gamma$, then taking $\gamma_{\lambda}=\gamma+\lambda \nu_{\Gamma}(\gamma)$ yields a parametrization of $\Gamma_{\lambda}$ and

$$
\dot{\gamma}_{\lambda}=\dot{\gamma}_{\Gamma}+\lambda \frac{d}{d t} \nu_{\Gamma}(\gamma)=[1+\lambda \kappa(\gamma)] \tau_{\Gamma}(\gamma),
$$

since $\frac{d}{d t} \nu_{\Gamma}(\gamma)=\kappa(\gamma) \tau_{\Gamma}(\gamma)$ for the curvature $\kappa$ along $\Gamma$. It follows that

$$
\frac{1}{c} \leq\left|\dot{\gamma}_{\lambda}\right|=|1+\lambda \kappa(\gamma)| \leq c, \lambda \in[-\Lambda, \Lambda]
$$

for some $c>1$ and $\Lambda<<1$. Consequently one has that

$$
\frac{1}{c} d x d y \leq d \sigma_{\Gamma} d \lambda \leq c d x d y .
$$

With this in hand, it follows that

$$
\begin{aligned}
\int_{\mathbb{B}(x, 1 / m)} \varphi_{m}(x-y) \frac{\alpha(x)}{\alpha(y)} d y & \sim m^{2} \int_{\Gamma \cap \mathbb{B}(x, 1 / m)} \int_{\lambda(x)-1 / m}^{\lambda(x)+1 / m} \frac{\alpha(y(x), \lambda(x))}{\alpha(\bar{y}, \bar{\lambda})} d \bar{\lambda} d \sigma_{\Gamma}(\bar{y}) \\
& \sim m \int_{\lambda(x)-1 / m}^{\lambda(x)+1 / m} \frac{|\lambda(x)|^{\sigma}}{|\bar{\lambda}|^{\sigma}} d \bar{\lambda}
\end{aligned}
$$


and the proof can be completed in a manner similar to that used in the one dimensional case by considering $x$ in distance-layers around $\Gamma$. The assumption on $\alpha$ yielding $\delta>0$ such that

$$
\alpha(x) \sim d(x, \Gamma)^{\sigma}=|\lambda(x)|^{\sigma} \text { in } T_{3 \delta}(\Gamma)
$$

was of course used in the above estimates.

Lemma 3.2 (Density). The space $\mathcal{D}_{\pi}(B)$ of periodic test-functions is dense in $\mathrm{H}_{\pi, \alpha}^{1}(B)$.

Proof. Let $u_{m}=\varphi_{m} * u$, so that $u_{m} \in \mathcal{D}_{\pi}(B)$, that

$$
u_{n} \rightarrow u \text { in } \mathrm{L}_{\pi}^{2}(B) \text { as } m \rightarrow \infty \text {. }
$$

and that

$$
\nabla u_{m} \rightarrow \nabla u \in \mathrm{L}_{\pi}^{1}(B)^{n} \text { as } m \rightarrow \infty
$$

for any $u \in \mathrm{H}_{\pi, \alpha}^{1}(B)$. Without loss of generality, it can be assumed that $\nabla u_{m} \rightarrow \nabla u$ pointwise almost everywhere (otherwise just take the appropriate subsequence). Then

$$
\alpha\left|\partial_{j} u_{m}\right|^{2} \longrightarrow \alpha\left|\partial_{j} u\right|^{2} \text { a.e. for } j=1,2 \text { as } m \rightarrow \infty \text {. }
$$

If it were possible to show that

$$
\alpha\left|\partial_{j} u_{m}\right|^{2} \leq g_{m}, m \in \mathbb{N},
$$

for nonnegative measurable functions $g_{m}$ which converge pointwise almost everywhere to $g \in \mathrm{L}_{\pi}^{1}(B)$ and for which

$$
\int_{B} g_{m} d x \longrightarrow \int_{B} g d x \text { as } m \rightarrow \infty,
$$

then the generalized Dominated Convergence Theorem would imply that

$$
\int_{B} \alpha\left|\partial_{j} u_{m}\right|^{2} d x \longrightarrow \int_{B} \alpha\left|\partial_{j} u\right|^{2} d x \text { as } m \rightarrow \infty
$$

which, together with the almost everywhere convergence, would yield

$$
\sqrt{\alpha} \nabla u_{m} \rightarrow \sqrt{\alpha} \nabla u \text { in } \mathrm{L}_{\pi}^{2}(B)^{n} \text { as } m \rightarrow \infty
$$

and the claim. Going back to (3.4), Lemma 3.1 gives

$$
\begin{array}{r}
\alpha(x)\left|\int_{B} \varphi_{m}(x-y) \partial_{j} u(y) d y\right|^{2} \leq \int_{B} \varphi_{m}(x-y) \frac{\alpha(x)}{\alpha(y)} d y \int_{B} \varphi_{m}(x-y) \alpha(y)\left|\partial_{j} u(y)\right|^{2} d y \\
\leq c \varphi_{m} *\left(\alpha\left|\partial_{j} u\right|^{2}\right), j=1,2, m \in \mathbb{N},
\end{array}
$$

and $u \in \mathrm{H}_{\pi, \alpha}^{1}(B)$ ensures that

$$
\varphi_{m} *\left(\alpha\left|\partial_{j} u\right|^{2}\right) \longrightarrow \alpha\left|\partial_{j} u\right|^{2} \text { in } \mathrm{L}_{\pi}^{1}(B) \text { as } m \rightarrow \infty,
$$

as desired.

Lemma 3.3 (Compact Embedding). The embedding $\mathrm{H}_{\pi, \alpha}^{1}(B) \hookrightarrow \mathrm{L}_{\pi}^{2}(B)$ is compact.

Proof. In view of assumption (2.1) on the weight function $\alpha$, an exponent $p>1$ can be found such that

$$
\int_{B} \frac{1}{\alpha(x)^{p}} d x<\infty .
$$

Then one has that $|\nabla u| \in \mathrm{L}_{\pi}^{1+\delta}(B)$ for some $\delta>0$ small enough since

$$
\begin{aligned}
\int_{B}|\nabla u(x)|^{1+\delta} d x \leq \int_{B}\left(\frac{\sqrt{\alpha(x)}}{\sqrt{\alpha(x)}}\right)^{1+\delta} \mid \nabla & \left.u(x)\right|^{1+\delta} d x \\
& \leq\left(\int_{B} \alpha(x)^{-\frac{1+\delta}{1-\delta}} d x\right)^{\frac{1-\delta}{2}}\left(\int_{B} \alpha(x)|\nabla u(x)|^{2} d x\right)^{\frac{1+\delta}{2}}<\infty
\end{aligned}
$$


provided $\frac{1+\delta}{1-\delta}<p$, which is always possible for a small enough $\delta$. This shows that $u \in \mathrm{W}_{\pi}^{1,1+\delta}(B)$ and the claim therefore follows from the compactness part of Sobolev embedding theorem observing that $2<(1+\delta)^{*}=\frac{n(1+\delta)}{n-1-\delta}$ is valid as long as $n<2 \frac{1+\delta}{1-\delta}$. This is always the case for dimensions $n=1,2$.

Lemma 3.4 (Existence of Traces). Any function $u \in \mathrm{H}_{\pi, \alpha}^{1}(B)$ admits a trace $\gamma_{\Gamma}(u) \in \mathrm{L}^{2}(\Gamma)$ on the degeneration set $\Gamma$.

Proof. Using the coordinates introduced in the proof of Lemma 3.1 for the tubular neighborhood $T_{\Lambda}(\Gamma)$ of $\Gamma$, take $u \in \mathcal{D}_{\pi}(B)$ and let $\left(\lambda_{m}\right)_{m \in \mathbb{N}}$ be a sequence in $(-\Lambda, \Lambda) \backslash\{0\}$ such that $\lambda_{m} \rightarrow 0$ as $m \rightarrow \infty$. Then

It follows that

$$
u\left(y, \lambda_{k}\right)-u\left(y, \lambda_{l}\right)=\int_{\lambda_{l}}^{\lambda_{k}} \partial_{\lambda} u(y, \lambda) d \lambda
$$

$$
\begin{aligned}
\left\|u\left(\cdot, \lambda_{k}\right)-u\left(\cdot, \lambda_{l}\right)\right\|_{L^{2}(\Gamma)} \leq \int_{\Gamma}\left|\int_{\lambda_{l}}^{\lambda_{k}} \partial_{\lambda} u(y, \lambda) d \lambda\right|^{2} d \sigma_{\Gamma}(y) \\
\quad \leq \int_{\Gamma}\left(\int_{\lambda_{l}}^{\lambda_{k}} \frac{1}{\alpha(y, \lambda)} d \lambda\right)\left(\int_{\lambda_{l}}^{\lambda_{k}} \alpha(y, \lambda)\left|\partial_{\lambda} u(y, \lambda)\right|^{2} d \lambda\right) d \sigma_{\Gamma}(y) .
\end{aligned}
$$

Noticing that

$$
d(x, \Gamma)=|\lambda(x)| \text { for } x \in T_{\Gamma}(\Lambda),
$$

assumption (2.1) on the diffusivity $\alpha$ now implies that

$$
\frac{1}{\alpha(y, \lambda)} \leq c \frac{1}{|\lambda|^{\sigma}},(y, \lambda) \in \Gamma \times(-\Lambda, \Lambda),
$$

for a constant $c$ independent of $(y, \lambda)$ and thus

$$
\int_{\lambda_{l}}^{\lambda_{k}} \frac{1}{\alpha(y, \lambda)} d \lambda \rightarrow 0 \text { as } k, l \rightarrow \infty
$$

As for the remaining integral one has

$$
\int_{\lambda_{l}}^{\lambda_{k}} \int_{\Gamma} \alpha(y, \lambda)\left|\partial_{\lambda} u(y, \lambda)\right|^{2} d \sigma_{\Gamma}(y) d \lambda \leq c \int_{T_{\Lambda}} \alpha|\nabla u|^{2} d x d y \leq c\|u\|_{\mathrm{H}_{\pi, \alpha}^{1}(B)}^{2},
$$

using (3.3) and that $\partial_{\lambda} u(y, \lambda)=\nabla u(y, \lambda) \cdot \nu_{\Gamma}(y) \leq|\nabla u(y, \lambda)|$. This shows that $\left(u\left(\cdot, \lambda_{m}\right)\right)_{m \in \mathbb{N}}$ is a Cauchy sequence in $\mathrm{L}^{2}(\Gamma)$ and thus that there exists a limit, which we denote by $\gamma_{\Gamma}(u) \in \mathrm{L}^{2}(\Gamma)$, such that

$$
\gamma_{\Gamma_{\lambda_{k}}}(u) \rightarrow \gamma_{\Gamma}(u) \text { as } k \rightarrow \infty .
$$

Observe that the trace operators $\gamma_{\Gamma_{\lambda_{k}}}$ are well-defined for any $k \in \mathbb{N}$ since $u \in \mathrm{H}^{1}\left(B \backslash T_{\varepsilon}(\Gamma)\right)$ for any $\varepsilon>0$ and $\Gamma_{\lambda_{k}} \subset B \backslash T_{\varepsilon}(\Gamma)$ for $\varepsilon<<1$. The construction of a trace for $u$ is therefore completed in the smooth case. The rest follows by the density established in Lemma 3.2

Lemma 3.5 (Poincaré Inequality). It holds that

$$
\|u\|_{\mathrm{L}_{\pi}^{2}(B)} \leq c\|\alpha \nabla u\|_{\mathrm{L}_{\pi}^{2}(B)}, u \in \mathrm{H}_{\pi, \alpha, 0}^{1}(B)
$$

where

$$
\mathrm{H}_{\pi, \alpha, 0}^{1}(B)=\left\{u \in \mathrm{H}_{\pi, \alpha}^{1}(B) \mid \int_{B} u(x) d x=0\right\} .
$$


Proof. Towards a contradiction assume that the inequality does not hold, that is, that there is a sequence $\left(u_{k}\right)_{k \in \mathbb{N}}$ in $\mathrm{H}_{\pi, \alpha, 0}^{1}(B)$ such that

$$
\left\|u_{k}\right\|_{\mathrm{L}_{\pi}^{2}(B)} \geq k\left\|\sqrt{\alpha} \nabla u_{k}\right\|_{\mathrm{L}_{\pi}^{2}(B)} .
$$

Define $v_{k}=u_{k} /\left\|u_{k}\right\|_{\mathrm{L}_{\pi}^{2}(B)}$ so that

$$
\left\|v_{k}\right\|_{\mathrm{L}_{\pi}^{2}(B)}=1 \text { and }\left\|\sqrt{\alpha} \nabla v_{k}\right\|_{\mathrm{L}_{\pi}^{2}(B)}=\frac{\left\|\sqrt{\alpha} \nabla u_{k}\right\|_{\mathrm{L}_{\pi}^{2}(B)}}{\left\|u_{k}\right\|_{\mathrm{L}_{\pi}^{2}(B)}} \leq \frac{1}{k}, k \in \mathbb{N} .
$$

In particular it holds that $\left\|v_{k}\right\|_{\mathrm{H}_{\pi, \alpha}^{1}(B)} \leq c<\infty$ for $k \in \mathbb{N}$ and, by the weak sequential compactness of bounded sets in Hilbert spaces, there must be $v_{\infty} \in \mathrm{H}_{\pi, \alpha, 0}^{1}(B)$ such that

$$
v_{k} \rightarrow v_{\infty} \text { in } \mathrm{H}_{\pi, \alpha, 0}^{1}(B) \text { along a subsequence. }
$$

The convergence of the norms then yields that $v_{k} \rightarrow v_{\infty}$ in $\mathrm{H}_{\pi, \alpha, 0}^{1}(B)$ along the subsequence. In this case $\nabla v_{\infty}=0$ almost everywhere in the two connected components $\Omega_{i}$ and $\Omega_{o}$ of $B \backslash \Gamma$ since, by weak lower semicontinuity, it holds that $\left\|\alpha \nabla v_{\infty}\right\|_{\mathrm{L}_{\pi}^{2}(B)} \leq \liminf \inf _{k \rightarrow \infty}\left\|\alpha \nabla v_{k}\right\|_{\mathrm{L}_{\pi}^{2}(B)}$ along the subsequence. Thus

$$
v_{\infty}(x)= \begin{cases}c_{i}, & x \in \Omega_{i} \\ c_{o}, & x \in \Omega_{o}\end{cases}
$$

and it can be inferred from Lemma 3.4 that necessarily $c_{i}=c_{o}$ since otherwise $v_{\infty}$ would not possibly possess a well-defined trace on $\Gamma$. The mean zero condition finally yields that the constant must be 0 . This clearly contradicts the fact that $\left\|v_{\infty}\right\|_{L_{\pi}^{2}(B)}=1$ and concludes the proof.

The above lemma clearly implies that $\|\sqrt{\alpha} \nabla \cdot\|_{\mathrm{L}_{\pi}^{2}(B)}$ is an equivalent norm on $\mathrm{H}_{\pi, \alpha}^{1}(B)$. The Poincaré inequality implies that the restriction of the nonnegative, continuous, and symmetric bilinear form (3.1) to $\mathrm{H}_{\pi, \alpha, 0}^{1}(B) \times \mathrm{H}_{\pi, \alpha, 0}^{1}(B)$ is elliptic and therefore induces a self-adjoint linear operator

such that

$$
\mathcal{A}_{\alpha, 0}: \mathrm{H}_{\pi, \alpha, 0}^{1}(B) \rightarrow \mathrm{H}_{\pi, \alpha, 0}^{1}(B)^{\prime}=: \mathrm{H}_{\pi, \alpha, 0}^{-1}(B), u \mapsto a_{\alpha}(u, \cdot),
$$

$$
\mathcal{A}_{\alpha, 0}: \mathrm{H}_{\pi, \alpha, 0}^{1}(B) \rightarrow \mathrm{H}_{\pi, \alpha, 0}^{-1}(B)
$$

is invertible and has, by Lemma 3.3 , compact resolvent. Here it holds that

$$
\mathrm{H}_{\pi, \alpha, 0}^{-1}(B)=\left\{u \in \mathrm{H}_{\pi, \alpha}^{1}(B)^{\prime} \mid\langle u, \mathbf{1}\rangle=0\right\}
$$

where 1 denotes the constant function with value 1 . It follows that

$$
\mathcal{A}_{\alpha}=\sum_{k=1}^{\infty} \mu_{k}\left(\cdot \mid \varphi_{k}\right) \varphi_{k}
$$

for $\left(\mu_{k}, \varphi_{k}\right)$ eigenvalue/eigenvector pairs of $A_{\alpha}$ with

$$
0<\mu_{1} \leq \mu_{2} \leq \ldots \mu_{k} \rightarrow \infty(k \rightarrow \infty),
$$

and where $\frac{1}{\sqrt{2^{n}}} \mathbf{1}=: \varphi_{0}, \varphi_{1}, \varphi_{2}, \ldots$ is an orthonormal basis for $\mathrm{H}_{\pi, \alpha}^{-1}(B)$. The $\mathrm{L}_{\pi}^{2}(B)$-realization $A_{\alpha}$ of $\mathcal{A}_{\alpha}$ will be particularly useful and is defined by $A_{\alpha} u=\mathcal{A}_{\alpha} u$ for

$$
\begin{aligned}
u \in \operatorname{dom}\left(A_{\alpha}\right) & =\left\{u \in \mathrm{H}_{\pi, \alpha}^{1}(B) \mid a_{\alpha}(u, \cdot) \text { is } \mathrm{L}_{\pi}^{2}(B) \text {-continuous }\right\} \\
& =\left\{u \in \mathrm{H}_{\pi, \alpha}^{1}(B) \mid \operatorname{div}(\alpha \nabla u) \in \mathrm{L}_{\pi}^{2}(B)\right\}=: \mathrm{H}_{\pi, \alpha}^{2}(B) .
\end{aligned}
$$

The second equality requires a proof. Assume that $\operatorname{div}(\alpha \nabla u) \in \mathrm{L}_{\pi}^{2}(B)$, then

$$
\int_{B} \alpha \underbrace{\nabla u}_{\in \mathrm{L}_{\pi, \alpha}^{2}(B)} \cdot \underbrace{\nabla v}_{\in \mathrm{L}_{\pi, \alpha}^{2}(B)} d x=-\int_{B} \underbrace{\operatorname{div}(\alpha \nabla u)}_{\in \mathrm{L}_{\pi}^{2}(B)} \underbrace{v}_{\in \mathrm{L}_{\pi}^{2}(B)} d x, v \in \mathcal{D}_{\pi}(B),
$$


and thus

Conversely, if

$$
\left|a_{\alpha}(u, v)\right| \leq\|\operatorname{div}(\alpha \nabla u)\|_{\mathrm{L}_{\pi}^{2}(B)}\|v\|_{\mathrm{L}_{\pi}^{2}(B)}, v \in \mathcal{D}_{\pi}(B)
$$

$$
\left|\int_{B} \alpha \nabla u \cdot \nabla v d x\right| \leq c\|v\|_{\mathrm{L}_{\pi}^{2}(B)}, v \in \mathcal{D}_{\pi}(B)
$$

then there is $w \in \mathrm{L}_{\pi}^{2}(B)$ such that

$$
\int_{B} \alpha \nabla u \cdot \nabla v d x=\int_{B} w v d x, v \in \mathcal{D}_{\pi}(B)
$$

which entails that $\operatorname{div}(\alpha \nabla u)=-w \in \mathrm{L}_{\pi}^{2}(B)$. Clearly $A_{\alpha}: \operatorname{dom}\left(A_{\alpha}\right) \subset \mathrm{L}_{\pi}^{2}(B) \rightarrow \mathrm{L}_{\pi}^{2}(B)$ is given by

$$
A_{\alpha} u=\sum_{k=1}^{\infty} \mu_{k} \underbrace{\left(u \mid \varphi_{k}\right)}_{:=\hat{u}_{k}} \varphi_{k}, u \in \operatorname{dom}\left(A_{\alpha}\right),
$$

and thus

$$
e^{-t A_{\alpha}} u=\hat{u}_{0}+\sum_{k=1}^{\infty} e^{-\mu_{k} t} \hat{u}_{k} \varphi_{k}, u \in \mathrm{L}_{\pi}^{2}(B)
$$

Notice that

$$
\begin{gathered}
\|u\|_{\mathrm{L}_{\pi}^{2}(B)}=\left\|\left(\hat{u}_{k}\right)_{k \in \mathbb{N}}\right\|_{l_{2}(\mathbb{N})} \text { and } \\
\left\|e^{-t A_{\alpha}} u\right\|_{L_{\pi}^{2}(B)}=\left\|\left(e^{-\mu_{k} t} \hat{u}_{k}\right)_{k \in \mathbb{N}}\right\|_{l_{2}(\mathbb{N})} \leq\left\|\left(\hat{u}_{k}\right)_{k \in \mathbb{N}}\right\|_{l_{2}(\mathbb{N})}=\|u\|_{\mathrm{L}_{\pi}^{2}(B)} .
\end{gathered}
$$

Thus $\left\{T_{1}(t):=e^{-t A_{\alpha}} \mid t \geq 0\right\}$ is a contraction semigroup and, since,

$$
\left\|t A_{\alpha} e^{-t A_{\alpha}} u\right\|_{\mathrm{L}_{\pi}^{2}(B)}=\left\|\left(t \mu_{k} e^{-\mu_{k} t} \hat{u}_{k}\right)_{k \in \mathbb{N}}\right\|_{l_{2}(\mathbb{N})} \leq c\|u\|_{\mathrm{L}_{\pi}^{2}(B)}, t>0
$$

it is also analytic (see [4]). Strong continuity can also be easily derived via the spectral representation (3.7). Summarizing

Theorem 3.6. The operators $A_{\alpha}$ and $\mathcal{A}_{\alpha}$ generate strongly continuous analytic contraction semigroups on $\mathrm{L}_{\pi}^{2}(B)$ and on $\mathrm{H}_{\pi, \alpha}^{-1}(B)$, respectively. In particular, for any given $u_{0} \in \mathrm{L}_{\pi}^{2}(B)\left[\mathrm{H}_{\pi, \alpha}^{-1}(B)\right]$, there is a unique solution $u \in \mathrm{C}\left([0, \infty), \mathrm{L}_{\pi}^{2}(B)\right)\left[\mathrm{C}\left([0, \infty), \mathrm{H}_{\pi, \alpha}^{-1}(B)\right)\right]$ of the abstract Cauchy problem

$$
\left\{\begin{array}{l}
\dot{u}=A_{\alpha} u \text { in } \mathrm{L}_{\pi}^{2}(B)\left[\dot{u}=\mathcal{A}_{\alpha} u \text { in } \mathrm{H}_{\pi, \alpha}^{-1}\right], \quad t>0, \\
u(0)=u_{0}
\end{array}\right.
$$

satisfying

$$
u \in \mathrm{C}^{1}\left((0, \infty), \mathrm{L}_{\pi}^{2}(B)\right) \cap \mathrm{C}\left((0, \infty), \mathrm{H}_{\pi, \alpha}^{2}(B)\right)\left[\mathrm{C}^{1}\left((0, \infty), \mathrm{H}_{\pi, \alpha}^{-1}(B)\right) \cap \mathrm{C}\left((0, \infty), \mathrm{H}_{\pi, \alpha}^{1}(B)\right)\right] .
$$

Moreover, one always has that

$$
u\left(t, u_{0}\right) \longrightarrow \frac{1}{2^{n}}\left\langle u_{0}, \mathbf{1}\right\rangle \text { as } t \rightarrow \infty,
$$

in $\mathrm{L}_{\pi}^{2}(B)\left[\mathrm{H}_{\pi, \alpha}^{-1}(B)\right]$.

Remark 3.7. Depending on the functional setting chosen, the above theorem yields a strong or weak solution of the initial boundary value problem

$$
\begin{cases}\dot{u}=\nabla \cdot(\alpha(x) \nabla u) & \text { in } B \text { for } t>0, \\ u(0, \cdot)=u_{0} & \text { in } B\end{cases}
$$

respectively. 
Remark 3.8. Notice how a piecewise constant initial datum is instanteneously regularized in spite of the fact that it is a steady-state of the equation. While the theorem ensures well-posedness in the specified classes of functions, the existence of additional solutions is observed also in numerical discretizations of the equation. More on this in Section 4.

Remark 3.9. While the semigroup is analytic, it does not follow that solutions are $\mathrm{C}^{\infty}$. This is due to the fact that the eigenfunctions are not smooth where the coefficient $\alpha$ vanishes.

3.2. The Singular Case. It was already noticed that $\widetilde{E}_{\alpha}$ has additional minimizers as compared to $E_{\alpha}$, for which only constant functions are minimizing. Let

$$
\mathbf{h}(x):= \begin{cases}\frac{1}{\sqrt{2^{n}}} \frac{|B \backslash \Omega|^{1 / 2}}{|\Omega|^{1 / 2}}, & x \in \Omega_{i}, \\ -\frac{1}{\sqrt{2^{n}}} \frac{|\Omega|^{1 / 2}}{|B \backslash \Omega|^{1 / 2}}, & x \in \Omega_{o},\end{cases}
$$

with the understanding that $\Omega_{i}=\Omega$ for $n=2, \Omega_{i}=[-1 / 2,1 / 2]$ for $n=1$, that $\Omega_{o}=B^{n} \backslash \Omega$ for $n=1,2$, and that $|S|$ is the Lebesgue measure of the measurable set $S$. Then $\mathbf{h}$ is a minimizer of $\widetilde{E}_{\alpha}$ and satisfies

$$
\int_{B} \mathbf{h}(x) d x=0 \text { and } \int_{B} \mathbf{h}^{2}(x) d x=1 .
$$

It is then possible to consider the modified energy functional

$$
\widetilde{\mathcal{E}}_{\alpha}(u, c):=\int_{B} \alpha|\nabla u|^{2} d x, u \in \mathrm{H}_{\pi, \alpha}^{1}(B), c \in \mathbb{R},
$$

on the space $\mathrm{H}_{\pi, \alpha}^{1}(B) \oplus \mathbb{R} \mathbf{h} \subset \mathrm{L}_{\pi}^{2}(B)$ and the associated gradient flow

$$
\left\{\begin{array}{l}
\dot{u}=\nabla \cdot(\alpha \nabla u) \\
\dot{c}=0 .
\end{array}\right.
$$

In this case, the solution $u$ to an initial value $u_{0}+c \mathbf{h} \in \mathrm{H}_{\pi, \alpha}^{1}(B) \oplus \mathbb{R} \mathbf{h}$, would satisfy

$$
u(t, \cdot) \longrightarrow \frac{1}{2^{n}} \int_{B} u_{0}(x) d x+c \mathbf{h},
$$

thus preserving the singular component during the entire evolution. While this is a perfectly acceptable interpretation of equation (2.3), it has some serious shortcomings. Most notably, the natural semigroup associated to it and given by

$$
T_{2}(t)=\left[\begin{array}{cc}
e^{-t A_{\alpha}} & 0 \\
0 & 1
\end{array}\right] \text { on } \mathrm{H}_{\pi, \alpha}^{1}(B) \oplus \mathbb{R} \mathbf{h}
$$

cannot be reasonably extended to $\mathrm{L}_{\pi}^{2}(B)$ as it is not $\mathrm{L}_{\pi}^{2}$-continuous as follows from

$$
\begin{aligned}
\left\|T_{2}(t)\left[\mathbf{h}_{m}-\mathbf{h}\right]\right\|_{2}=\left\|e^{-t A_{\alpha}} \mathbf{h}_{m}-\mathbf{h}\right\|_{2} & =\left\|\sum_{k=1}^{m} e^{-t \lambda_{k}} \hat{\mathbf{h}}_{k} \varphi_{k}-\sum_{k=1}^{\infty} \hat{\mathbf{h}}_{k} \varphi_{k}\right\|_{2} \\
& \longrightarrow\left[\sum_{k=1}^{\infty}\left(1-e^{-t \lambda_{k}}\right)^{2} \hat{\mathbf{h}}_{k}^{2}\right]^{1 / 2} \neq 0 \text { for any } t>0 \text { as } m \rightarrow \infty,
\end{aligned}
$$

where

$$
\mathrm{H}_{\pi, \alpha}^{1}(B) \ni \mathbf{h}_{m}:=\sum_{k=1}^{m} \hat{\mathbf{h}}_{k} \varphi_{k} \longrightarrow \mathbf{h} \text { in } \mathrm{L}_{\pi}^{2}(B) \text { as } m \rightarrow \infty
$$


3.3. The Split Case. Given the diffusion coefficient $\alpha \in \mathrm{D}_{\pi}^{\sigma}$, one can consider the energy functional

$$
\bar{E}_{\alpha}\left(u^{i}, u^{o}\right)=\frac{1}{2} \int_{\Omega^{i}} \alpha\left|\nabla u^{i}\right|^{2} d x+\frac{1}{2} \int_{\Omega^{o}} \alpha\left|\nabla u^{o}\right|^{2} d x,\left(u^{i}, u^{o}\right) \in \mathrm{H}_{\alpha}^{1}\left(\Omega^{i}\right) \times \mathrm{H}_{\alpha, \pi}^{1}\left(\Omega^{o}\right),
$$

where $\Omega^{i}$ and $\Omega^{o}$ have previously been defined. Arguments perfectly analogous to those used in Section 3.1 can be used to prove the following result.

Theorem 3.10. The restriction of the functional $\bar{E}_{\alpha}$ to $\mathrm{H}_{\alpha, 0}^{1}\left(\Omega^{i}\right) \times \mathrm{H}_{\alpha, \pi, 0}^{1}\left(\Omega^{o}\right) \rightarrow \mathbb{R}$ is coercive and the operator induced by $\bar{E}_{\alpha}$

$$
\mathcal{A}_{\alpha}=\operatorname{diag}\left(\mathcal{A}_{\alpha}^{i}, \mathcal{A}_{\alpha}^{o}\right): \mathrm{H}_{\alpha}^{1}\left(\Omega^{i}\right) \times \mathrm{H}_{\alpha, \pi}^{1}\left(\Omega^{o}\right) \rightarrow \mathrm{H}_{\alpha}^{-1}\left(\Omega^{i}\right) \times \mathrm{H}_{\alpha, \pi}^{-1}\left(\Omega^{o}\right)
$$

and

$$
A_{\alpha}=\operatorname{diag}\left(A_{\alpha}^{i}, A_{\alpha}^{o}\right): \operatorname{dom}\left(A_{\alpha}^{i}\right) \times \operatorname{dom}\left(A_{\alpha}^{o}\right) \rightarrow \mathrm{L}^{2}\left(\Omega^{i}\right) \times \mathrm{L}_{\pi}^{2}\left(\Omega^{o}\right) \hat{=} \mathrm{L}_{\pi}^{2}(B)
$$

with

$$
\operatorname{dom}\left(A_{\alpha}^{l}\right)=\left\{u \in \mathrm{L}^{2}\left(\Omega^{l}\right) \mid \operatorname{div}(\alpha \nabla u) \in \mathrm{L}^{2}\left(\Omega^{l}\right)\right\}, l=i, o .
$$

generate analytic contraction semigroups on $\mathrm{H}_{\alpha}^{-1}\left(\Omega^{i}\right) \times \mathrm{H}_{\alpha, \pi}^{-1}\left(\Omega^{o}\right)$ and on $\mathrm{L}_{\pi}^{2}(B)$, repectively. Call the latter $T_{3}(t)$. It follows that the system

$$
\begin{cases}u_{t}^{i}=\nabla \cdot\left(\alpha \nabla u^{i}\right) & \text { in } \Omega^{i} \text { for } t>0, \\ u_{t}^{o}=\nabla \cdot\left(\alpha \nabla u^{o}\right) & \text { in } \Omega^{o} \text { for } t>0, \\ \lim _{x \rightarrow \Gamma} \alpha(x) \partial_{\nu_{\Gamma}} u^{i}(x)=0 & \\ \lim _{x \rightarrow \Gamma} \alpha(x) \partial_{\nu_{\Gamma}} u^{o}(x)=0 & \\ u^{i}(0, \cdot)=u_{0}^{i} & \text { in } \Omega^{i} \\ u^{o}(0, \cdot)=u_{0}^{o} & \text { in } \Omega^{o}\end{cases}
$$

is uniquely (weakly) solvable for any $u_{0} \in \mathrm{L}_{\pi}^{2}(B)$ (or, more in general, for an initial datum $u_{0} \in$ $\left.\mathrm{H}_{\alpha}^{-1}\left(\Omega^{i}\right) \times \mathrm{H}_{\alpha, \pi}^{-1}\left(\Omega^{o}\right)\right)$, and the solution converges to a trivial steady-state in each subdomain, that is,

$$
T_{3}(t) u_{0} \longrightarrow\left(\frac{1}{\left|\Omega_{i}\right|} \int_{\Omega_{i}} u_{0}(x) d x\right) \chi_{\Omega_{i}}+\left(\frac{1}{\left|\Omega_{o}\right|} \int_{\Omega_{o}} u_{0}(x) d x\right) \chi_{\Omega_{o}}
$$

for $u_{0} \in \mathrm{L}_{\pi}^{2}(\Omega)$.

In this interpretation, one obtains an evolution on $\mathrm{L}_{\pi}^{2}(B)$ for which an initial datum that is constant on each of the domains $\Omega^{l}, l=i, o$, is a stationary solution and won't be regularized nor evolved.

Remark 3.11. Taking the system point of view, it is possible to recover the interpretation of Section 1 by defining the energy functional $\bar{E}_{\alpha}$ on

$$
\left\{u=\left(u^{i}, u^{o}\right) \mid u \in \mathrm{H}_{\alpha}^{1}\left(\Omega^{i}\right) \times \mathrm{H}_{\alpha, \pi}^{1}\left(\Omega^{o}\right) \text { and } \gamma_{\Gamma}\left(u^{i}\right)=\gamma_{\Gamma}\left(u^{o}\right)\right\} .
$$

This means that "continuity" across the interface has to be explicitly enforced.

Remark 3.12. Notice that the behavior of solutions in this interpretation is possibily what one would like to see from an application to image processing point of view in that solutions not only tend to become piecewise constant but the constants are also the local averages of the initial datum in the corresponding regions of constancy. 


\section{A Numerical Remark}

The non-uniqueness phenomenon highlighted above will be investigated for a spatial semidiscretization of (2.2) in a one-dimensional setting. The observation extends to the two-dimensional setting with the appropriate modifications. It is observed from the outset that, even the same numerical scheme, can produce two distinct solutions. One is the discrete counterpart of the regularizing solution of Section 3.1 the other "feels" the presence of the singular solution $h$ but, interestingly, is not the compatible with any of the three interpretations of equation (2.3) given above. An explanation of its origin will follow in the later part of this section.

Letting $n=1$ and $\alpha \in D_{\pi}^{\sigma}$ as in the previous sections and choosing

$$
\mathbf{h}=\frac{1}{\sqrt{2}} \chi_{\Omega_{i}}-\frac{1}{\sqrt{2}} \chi_{\Omega_{o}}
$$

Theorem 3.6 yields a solution

$$
u \in \mathrm{C}\left([0, \infty), \mathrm{L}_{\pi}^{2}(-1,1)\right) \cap \mathrm{C}^{1}\left((0, \infty), \mathrm{L}_{\pi}^{2}(-1,1)\right) \cap \mathrm{C}\left((0, \infty), \mathrm{H}_{\pi, \alpha}^{2}(-1,1)\right),
$$

for

where

$$
\left\{\begin{array}{l}
\dot{u}=\partial_{x}\left(\alpha(x) \partial_{x} u\right) \quad \text { in }(-1,1) \text { for } t>0 \\
u \text { periodic } \\
u(0, \cdot)=\mathbf{h}
\end{array}\right.
$$

$$
\mathrm{H}_{\pi, \alpha}^{2}(-1,1)=\left\{u \in \mathrm{H}_{\pi, \alpha}^{1}(-1,1) \mid \alpha u^{\prime} \in \mathrm{H}_{\pi}^{1}(-1,1)\right\}
$$

as follows from characterization (3.5) of the previous section. Theorem 3.6 then implies that

$$
u(t, \cdot)=T_{1}(t) \mathbf{h} \rightarrow \frac{1}{2} \int_{-1}^{1} \mathbf{h}(x) d x=0 \text { as } t \rightarrow \infty .
$$

It, however, also holds that

$$
\alpha \mathbf{h}^{\prime}=\alpha\left(\delta_{-1 / 2}-\delta_{1 / 2}\right)=\alpha(-1 / 2) \delta_{-1 / 2}-\alpha(1 / 2) \delta_{1 / 2}=0,
$$

so that $u(t, \cdot) \equiv u_{0}$ is a stationary solution of (4.1). This non-uniqueness is reflected at the numerical level. Indeed set

$$
\begin{aligned}
x_{i}^{m} & =\frac{i}{m}, i=-m,-m+1, \ldots, m-1, m, \\
d_{m} & =1 / m, \\
\alpha_{i}^{m} & =\alpha\left(x_{i}^{m}\right) .
\end{aligned}
$$

Then

$$
u_{t}^{m}=\Delta^{m,-}\left(\alpha^{m} \Delta^{m,+}\left(u^{m}\right)\right)
$$

is the gradient flow to the discrete energy functional given by

$$
E_{\alpha}^{m}\left(u^{m}\right)=\frac{1}{2} \sum_{i=-m}^{m-1}\left[\alpha_{i}^{m} \Delta_{i}^{m,+}\left(u^{m}\right)\right]^{2} d_{m}
$$

where

$$
\begin{aligned}
& \Delta_{i}^{m,+}\left(u^{m}\right)=\frac{u_{i+1}^{m}-u_{i}^{m}}{d_{m}}, i=-m, \ldots, m-1, \\
& \Delta_{i}^{m,-}\left(u^{m}\right)=\frac{u_{i}^{m}-u_{i-1}^{m}}{d_{m}}, i=-m, \ldots, m-1,
\end{aligned}
$$

with the understanding that

$$
u_{-m-1}^{m}=u_{m-1}^{m} \text { and that } u_{m+1}^{m}=u_{-m+1}^{m},
$$


enforcing periodicity. The ordinary differential equation (4.2) is a spatial semi-discretization of (4.1), and (4.3) is one of the continuous energy functional (2.4) on $\mathrm{H}_{\pi, \alpha}^{1}(-1,1)$. This is seen by computing

$$
\begin{aligned}
& \left.\frac{d}{d \epsilon}\right|_{\epsilon=0} E_{\alpha}^{m}\left(u^{m}+\epsilon \varphi^{m}\right)=\sum_{i=-m}^{m-1} \alpha_{i}^{m} \Delta_{i}^{m,+}\left(\varphi^{m}\right) \Delta_{i}^{m,+}\left(u^{m}\right) d_{m} \\
& \quad=-\sum_{i=-m}^{m-1}\left[\alpha_{i}^{m} \frac{u_{i+1}^{m}-u_{i}^{m}}{d_{m}}-\alpha_{i-1}^{m} \frac{u_{i}^{m}-u_{i-1}^{m}}{d_{m}}\right] \varphi_{i}^{m} d_{m}=-\sum_{i=-m}^{m-1} \Delta_{i}^{m,-}\left[\alpha^{m} \Delta^{m,+}\left(u^{m}\right)\right] \varphi_{i}^{m} d_{m} .
\end{aligned}
$$

Using test-vectors $\varphi^{m}=\frac{1}{d_{m}} e_{i}^{m}$ where $e_{i}^{m} \in \mathbb{R}^{m}$ is the $i$-th natural basis vector (which satisfies $\varphi_{i}^{m} \rightarrow \delta_{x}$ if $\frac{i}{m} \rightarrow x$ as $m \rightarrow \infty$ ) yields

$$
\dot{u}^{m}=-\nabla E_{\alpha}^{m}\left(u^{m}\right)=\Delta^{m,-}\left[\alpha^{m} \Delta^{m,+}\left(u^{m}\right)\right] .
$$

Notice that

$$
\begin{aligned}
2 \frac{d}{d t} \operatorname{avg}\left(u^{m}\right)=\frac{d}{d t} \sum_{i=-m}^{m-1} & u_{i}^{m} d_{m}=\sum_{i=-m}^{m-1} \dot{u}_{i}^{m} d_{m} \\
& =\sum_{i=-m}^{m-1} \Delta_{i}^{m,-}\left(\alpha^{m} \Delta^{m,+}\left(u^{m}\right) d_{m}=-\sum_{i=-m}^{m-1} \alpha_{i}^{m} \Delta_{i}^{m,+}(u) \Delta_{i}^{m,+}(\mathbf{1}) d_{m}=0\right.
\end{aligned}
$$

for $t \geq 0$. This shows that constant vectors are in the kernel $\nabla E_{\alpha}^{m}$ and thus minimizers of $E_{\alpha}^{m}$.

When $m$ is odd, these are the only minimizers of zero energy since

$$
\alpha_{i}^{m} \geq \min _{j=-m, \ldots, m-1} \alpha\left(x_{j}^{m}\right) \simeq\left(\frac{d_{m}}{2}\right)^{\sigma}>0,
$$

and, consequently, $\Delta^{m,+}\left(u^{m}\right) \equiv 0$ for any minimizer $u^{m}$. Thus, for odd $m$, one has that

$$
u^{m}(t) \rightarrow \frac{1}{2} \sum_{i=-m}^{m-1} u_{0}^{m} d_{m}=\operatorname{avg}\left(u_{0}^{m}\right) \text { as } t \rightarrow \infty,
$$

if $u_{0}^{m}$ is the initial vector, just as for $T_{1}(t) u_{0}$ at the continuous level. On the other hand, when $m$ is even, vectors $H^{m}\left(c_{1}, c_{2}\right)$ defined by

$$
H^{m}\left(c_{1}, c_{2}\right)= \begin{cases}c_{1}, & -m / 2<i \leq m / 2 \\ c_{2}, & i>m / 2 \text { and } i \leq-m / 2\end{cases}
$$

for any constants $c_{1}$ and $c_{2}$ also possess zero energy since $\alpha_{ \pm m / 2}^{m}=0$. In this case

$$
u^{m}(t) \rightarrow \operatorname{avg}\left(u_{0}^{m}\right)+\frac{d_{m}}{2}\left[H^{m}(1,-1) \cdot u_{0}^{m}\right] H^{m}(1,-1)
$$

This shows that two distinct solutions can be obtained numerically and that $E_{\alpha}^{m}$ does not have a well-defined unique gradient flow associated to it as $m \rightarrow \infty$ since the evolution clearly depends on the parity of $m$. Notice also that the large time behavior of $u^{m}$ is, for general initial data, incompatible with that of $T_{2}$ and $T_{3}$ as well, since

$$
T_{2}(t)\left(v_{0}+c \mathbf{h}\right) \longrightarrow \frac{1}{2} \int_{-1}^{1} v_{0}(x) d x+c \mathbf{h} \text { as } t \rightarrow \infty,
$$

and

$$
T_{3}(t)\left(v_{0}+c \mathbf{h}\right) \longrightarrow\left(\int_{\Omega_{i}} v_{0}(x) d x+c\right) \chi_{\Omega_{i}}+\left(\int_{\Omega_{o}} v_{0}(x) d x-c\right) \chi_{\Omega_{o}} \text { as } t \rightarrow \infty,
$$


if $u_{0}=v_{0}+c \mathbf{h}$. Limit (4.4) is in general not the discrete counterpart of any of these latter limits. As it turns out, the behavior of the above discretization, is compatible with the behavior of solutions of strongly degenerate equations. To see that, assume that $\alpha$ is a periodic functions, which is Hölder continuous of a positive exponent, positive everywhere away from $\pm \frac{1}{2}$ and satisfies

$$
\alpha(x) \sim\left|x \pm \frac{1}{2}\right|^{1+\sigma} \text { as } x \simeq \mp \frac{1}{2},
$$

for some $\sigma>0$. It follows that $\frac{1}{\alpha} \notin \mathrm{L}_{\pi}^{1}$ and that (2.2) is strongly degenerate. Define again

$$
\mathrm{H}_{\pi, \alpha}^{1}(B)=\left\{u \in \mathrm{L}_{\pi}^{2}(B) \mid \sqrt{\alpha} u^{\prime} \in \mathrm{L}_{\pi}^{2}(B)\right\},
$$

and notice, that now, not only $\mathbf{h} \in \mathrm{H}_{\pi, \alpha}^{1}(B)$ but also that

$$
\int_{-1}^{1} \alpha\left|\left(\varphi_{m} * \mathbf{h}\right)^{\prime}(x)\right|^{2} d x \rightarrow\left\|\sqrt{\alpha} \mathbf{h}^{\prime}\right\|_{2}^{2}=0 \text { as } m \rightarrow \infty .
$$

This shows that, in the strongly degenerate case, the space

$$
\left\{u \in \mathrm{L}_{\pi}^{2}(B) \mid u^{\prime} \in \mathrm{L}_{\pi}^{1}(B) \text { and } \sqrt{\alpha} u^{\prime} \in \mathrm{L}_{\pi}^{2}(B)\right\}
$$

is not closed and can therefore not be viewed as the "natural" domain of the energy functional $E_{\alpha}$ as in the weakly degenerate case. Let

$$
\mathrm{H}_{\pi, \alpha, 0}^{1}(B)=\left\{u \in \mathrm{H}_{\pi, \alpha}^{1}(B) \mid\langle u, \mathbf{1}\rangle=0,\langle u, \mathbf{h}\rangle=0\right\}
$$

Lemma 4.1. It holds that

$$
\int_{-1}^{1}|u(x)|^{2} d x \leq c \int_{-1}^{1} \alpha(x)\left|u^{\prime}(x)\right|^{2} d x \text { for } u \in \mathrm{H}_{\pi, \alpha, 0}^{1}(B) .
$$

Proof. Assume that this is not the case. Then a sequence $\left(u_{k}\right)_{k \in \mathbb{N}}$ in $\mathrm{H}_{\pi, \alpha, 0}^{1}(B)$ can be found such that

$$
1=\left\|u_{k}\right\|_{2}^{2} \geq k\left\|\sqrt{\alpha} u_{k}^{\prime}\right\|_{2}^{2}, k \in \mathbb{N} .
$$

It follows that $\sqrt{\alpha} u_{k}^{\prime} \longrightarrow 0$ in $\mathrm{L}_{\pi}^{2}(B)$. Now let $B_{\varepsilon}=\left[\left|x \pm \frac{1}{2}\right| \geq \varepsilon\right]$ for small $\varepsilon>0$. Then

$$
\left\|\chi_{B_{\varepsilon}} u_{k}^{\prime}\right\|_{2}^{2} \leq c\left\|\sqrt{\alpha} u^{\prime}\right\|_{2}^{2} \leq \frac{c}{k}, k \in \mathbb{N} \text {. }
$$

It follows that $\chi_{B_{\varepsilon}} u_{k} \rightarrow 0$ as $k \rightarrow \infty$ (along a subsequence) in $\mathrm{H}_{\pi}^{1}\left(B_{\varepsilon}\right)$ and therefore that

$$
\chi_{B_{\varepsilon}} u^{\prime}=0 \text { a.e. }
$$

for any small $\varepsilon>0$. It follows that $u$ must be constant on $\Omega_{i}$ and on $\Omega_{o}$ and, consequently, that

$$
\operatorname{supp}\left(u^{\prime}\right) \subset\{-1 / 2,1 / 2\} .
$$

Since $u \in \mathrm{L}_{\pi}^{2}(B)$, the distributional derivative $u^{\prime}$ has at most order 1 as follows from

$$
\left|\left\langle u^{\prime}, \varphi\right\rangle\right|=\left|\left\langle u, \varphi^{\prime}\right\rangle\right| \leq\|u\|_{2}\left\|\varphi^{\prime}\right\|_{2} \leq c\|u\|_{2}\left\|\varphi^{\prime}\right\|_{\infty}, \varphi \in \mathcal{D}_{\pi}(B) .
$$

Combining this with the support condition above, it is concluded that

$$
u^{\prime}=A \delta_{-1 / 2}+B \delta_{1 / 2}+C \delta_{-1 / 2}^{\prime}+D \delta_{1 / 2}^{\prime}
$$

for some constants $A, B, C, D$. Since $u \in \mathrm{L}_{\pi}^{2}(B)$, it must then hold that $C=D=0$. One also has that

$$
A+B=\left\langle u^{\prime}, \mathbf{1}\right\rangle=-\langle u, \mathbf{0}\rangle=0,
$$

and consequently that $u=\tilde{A}+\tilde{B} \mathbf{h}$ for some constants $\tilde{A}$ and $\tilde{B}$, which must both vanish since $u \in \mathrm{H}_{\pi, \alpha, 0}^{1}(B)$. This clearly yields a contradiction to $\|u\|_{2}=1$. 
The form

$$
a_{\alpha}(u, v)=\frac{1}{2} \int_{-1}^{1}\left(\sqrt{\alpha} u^{\prime}\right)(x)\left(\sqrt{\alpha} v^{\prime}\right)(x) d x
$$

defined on $\mathrm{H}_{\pi, \alpha, 0}^{1}(B) \times \mathrm{H}_{\pi, \alpha, 0}^{1}(B)$ is therefore coercive and the associated operator $\mathcal{A}_{\alpha}$ invertible. The solution $u$ of the corresponding heat equation (2.3) with initial datum $u_{0}$ therefore satisfies

$$
u\left(t, u_{0}\right) \longrightarrow \frac{1}{2}\left\langle u_{0}, \mathbf{1}\right\rangle+\left\langle u_{0}, \mathbf{h}\right\rangle \mathbf{h} \text { as } t \rightarrow \infty,
$$

just as the numerical solution when $m$ is even. It can be concluded that, at the discrete level, the distinction between weakly and strongly degenerate equations can go lost in certain cases.

Remark 4.2. Observe that it is more likely (especially in higher dimensions) that a numerical scheme will deliver the "smooth" solution of the continuous equation rather than the stationary one (for piecewise constant initial data). This is due to the fact that latter solution can only be captured if the jumps are on (or close enough) to the grid and Dirac delta functions at the jump locations discretize to discrete delta functions (read natural basis vectors). This is the case in the above example when $m$ is even but could not hold, e.g., for a centered difference scheme based on discretizing the first derivative by

i.e. for

$$
\Delta_{i}^{m, c}\left(u^{m}\right)=\frac{u_{i+1}^{m}-u_{i-1}^{m}}{2 h_{n}}, i=-m, \ldots, m-1,
$$

$$
\widetilde{E}_{\alpha}^{m}\left(u^{m}\right)=\frac{1}{2} \sum_{i=-m}^{m-1} \alpha_{i}^{m}\left[\frac{u_{i+1}^{m}-u_{i-1}^{m}}{2 h_{n}}\right]^{2} h_{n} .
$$

That said, the above example is not pathological. Indeed spectral discretizations in combination with appropriate discrete quadrature rules for the discretization of integrals (duality pairings) also capture the "singular" rather than the regular solution. This follows again from the fact that continuous delta functions discretize to discrete delta functions as is proved in [5].

\section{Regularization}

Next it is shown that the regularizing interpretation of (2.3) can be view as the limit of the regularized problem

$$
\begin{cases}\dot{u}=\nabla \cdot([1 / m+\alpha] \nabla u) & \text { in } B \text { for } t>0, \\ u(0)=u_{0} & \text { in } B .\end{cases}
$$

as $m \rightarrow \infty$. Start with the regularized energy functional

$$
E_{\alpha}^{m}(u):= \begin{cases}\int_{B}[1 / m+\alpha]|\nabla u|^{2} d x, & u \in \mathrm{H}_{\pi}^{1}(B), \\ \infty, & u \in \mathrm{L}_{\pi}^{2}(B) \backslash \mathrm{H}_{\pi}^{1}(B) .\end{cases}
$$

Proposition 5.1. It holds that $E_{\alpha}^{m} \stackrel{\Gamma}{\longrightarrow} E_{\alpha}$ (where $E_{\alpha}$ is extended by $\infty$ to $\mathrm{L}_{\pi}^{2}(B) \backslash \mathrm{H}_{\pi, \alpha}^{1}(B)$ ) as $m \rightarrow \infty$ with respect to the weak topology of $\mathrm{H}_{\pi, \alpha}^{1}(B)$.

Remark 5.2. The reason to consider $\Gamma$-convergence is that the domain of definition of the energy functional changes in the limit. As a consequence convergence can only be proved in a topology that is too weak to preserve the equation.

Proof. Following e.g. 2], $\Gamma$-convergence (with respect with the weak topology) is defined by the validity of the following estimates

(i) $E_{\alpha}(u) \leq \liminf _{m \rightarrow \infty} E_{\alpha}^{m}\left(u_{m}\right)$ for any $\mathrm{H}_{\pi}^{1}(B) \ni u_{m} \rightarrow u$ in $\mathrm{H}_{\pi, \alpha}^{1}(B)$

(ii) For any $u \in \mathrm{H}_{\pi, \alpha}^{1}(B)$ there is $\left(u_{m}\right)_{m \in \mathbb{N}}$ in $\mathrm{H}_{\pi}^{1}(B), u_{m} \rightarrow u$, with $E_{\alpha}(u)=\lim _{n \rightarrow \infty} E_{\alpha}^{m}\left(u_{m}\right)$. 
Let $\left(u_{m}\right)_{m \in \mathbb{N}}$ be any sequence in $\mathrm{H}_{\pi}^{1}(B)$ converging to $u \in \mathrm{H}_{\pi, \alpha}^{1}$ in the weak topology of the latter space. Then it clearly holds that

$$
E_{\alpha}\left(u_{m}\right) \leq E_{\alpha}^{m}\left(u_{m}\right), m \in \mathbb{N}
$$

and thus

$$
E_{\alpha}(u) \leq \liminf _{m \rightarrow \infty} E_{\alpha}\left(u_{m}\right) \leq \liminf _{m \rightarrow \infty} E_{\alpha}^{m}\left(u_{m}\right)
$$

since the first inequality follows from the weak lower semicontinuity of the norm on the Hilbert space $\mathrm{H}_{\pi, \alpha}^{1}(B)$. In order to verify the second condition, let $\varphi_{m}$ be the mollifier introduced immediately preceding the formulation of Lemma 3.1. It will be shown that

$$
\int_{B}[\alpha(x)+1 / m]\left|\nabla u^{m}(x)\right|^{2} d x \rightarrow \int_{B} \alpha|\nabla u|^{2} d x
$$

as $m \rightarrow \infty$ for $u^{m}:=\varphi_{m} * u \in \mathrm{H}_{\pi}^{1}(B)$. It is a consequence of Lemma 3.2 that

$$
\int_{B} \alpha(x)\left|\nabla u^{m}(x)\right|^{2} d x \rightarrow \int_{B} \alpha|\nabla u|^{2} d x .
$$

To deal with the second term, notice that

$$
\left[\int_{B} \varphi_{m}(\cdot-\bar{x}) \partial_{j} u(\bar{x}) d \bar{x}\right]^{2} \leq \int_{B} \frac{\varphi_{m}(\cdot-\bar{x})}{\alpha(\bar{x})} d \bar{x} \int_{B} \varphi_{m}(\cdot-\bar{x}) \alpha(\bar{x})\left(\partial_{j} u(\bar{x})\right)^{2} d \bar{x} .
$$

As the second factor on the right-hand-side converges to $\alpha\left|\partial_{j} u\right|^{2}$ in $\mathrm{L}_{\pi}^{1}(B)$ and the first can be estimated as follows

$$
\begin{array}{r}
\int_{B} \frac{\varphi_{m}(\cdot-\bar{x})}{\alpha(\bar{x})} \leq m^{2} \int_{\mathbb{B}(x, 1 / m)} \frac{d \bar{x}}{\alpha(\bar{x})}=m^{2}\left\{\int_{\mathbb{B}(x, 1 / m) \cap T_{1 / m}(\Gamma)^{c}}+\int_{\mathbb{B}(x, 1 / m) \cap T_{1 / m}(\Gamma)}\right\} \frac{d \bar{x}}{\alpha(\bar{x})} \\
\leq c m^{2} \int_{\mathbb{B}(x, 1 / m)} m^{\sigma} d \bar{x}+c m^{2} \int_{-1 / m}^{1 / m} \int_{\Gamma \cap \mathbb{B}(x, 1 / m)} \frac{1}{|\bar{s}|^{\sigma}} d \sigma_{\Gamma}(\bar{y}) d \bar{s}=c m^{\sigma}
\end{array}
$$

it can be concluded that

$$
\frac{1}{m} \int_{B}\left[\int_{B} \varphi_{m}(x-\bar{x}) \partial_{j} u(\bar{x}) d \bar{x}\right]^{2} d x \leq c m^{\sigma-1}\left\|\varphi_{m} *\left(\alpha\left|\partial_{i} u\right|^{2}\right)\right\|_{1} \longrightarrow 0 \text { as } m \rightarrow \infty .
$$

The proof is complete.

In spite of the fact that both the regularized problem and the limiting one generate analytic semigroups, solutions of the first do not converge to solutions of the latter in any strong way. This is due to the loss of regularity in the limit, where eigenfunctions (and, more in general, solutions) are no longer smooth (on the degeneration set). In view of Proposition 5.1 , however, $\Gamma$-convergence proves a useful tool for the purpose. In fact, known results for gradient flows showing that, if a sequence of energies $\Gamma$-converges to a limiting energy, so do the minimizing movements of the corresponding gradient flows, apply and yield a convergence result. Minimizing movements $u$ for a, in this context, convex energy functional $E$ on a Hilbert space $H$ are constructed as (locally uniform) limits

$$
u(t)=\lim _{h \rightarrow 0+} u^{h}(t),
$$

of approximating piecewise constant functions $u^{h}(t)=u^{h,\lfloor t / h\rfloor}$ obtained by recursive minimization

$$
u^{h, k+1}=\arg \min _{v \in H}\left\{E(v)+\frac{1}{2 h}\left\|v-u^{h, k}\right\|_{H}^{2}\right\}
$$

starting from an initial datum $u_{0}^{h}$. Latter essentially amounts to solutions of the Euler scheme with time step $h>0$ for the corresponding gradient flow. 
Remark 5.3. Observe that, when $E$ is a quadratic and therefore differentiable functional, and the linear operator $\mathcal{A}=\nabla E$ is the generator of a strongly continuous analytic semigroup of contractions as is the case for $E_{\alpha}^{m}$ and $E_{\alpha}$, then the minimization problem (5.3) is equivalent to

$$
(1+h \mathcal{A}) v=u^{h, k} \text { in } \mathrm{H}_{\pi}^{-1}(B) \text { or } \mathrm{H}_{\pi, \alpha}^{-1}(B),
$$

for $\mathcal{A}=\mathcal{A}_{\alpha}^{m}$ or $\mathcal{A}=\mathcal{A}_{\alpha}$ respectively. Consequently, one has that

$$
u^{h, k}=(1+h \mathcal{A})^{-1} u^{h, k-1}=(1-h \mathcal{A})^{-k} u_{0}^{h} .
$$

When $u_{0}^{h}=u_{0} \in \mathrm{L}_{\pi}^{2}(B)$ and $k h \rightarrow t$, semigroup theory (see [4) implies that

$$
(1+h \mathcal{A})^{-k} u_{0} \rightarrow e^{-t \mathcal{A}} u_{0}=T_{1}(t) u_{0} \text { as } h \rightarrow 0 .
$$

In this case, the minimizing movement originating in $u_{0}$ coincides with the solution that was previously constructed by the semigroup approach.

The following theorem is stated and proved in [2, Chapter 11].

Theorem 5.4. Let $\left(F_{m}\right)_{m \in \mathbb{N}}$ be a sequence of equi-coercive, lower semicontinuous, positive convex energies $\Gamma$-converging to $F$, and let $x_{0}^{m} \rightarrow x_{0}$ with $\sup _{m \in \mathbb{N}} F^{m}\left(x_{0}^{m}\right)<\infty$. Then the sequence of minimizing movement $u_{m}$ for $F^{m}$ starting in $x_{0}^{m}$ converges to the minimizing movement $u$ for $F$ originating in $x_{0}$.

This theorem yields the following result in the situation considered in this paper.

Theorem 5.5. Let $\mathrm{H}_{\pi}^{1}(B) \ni u_{0}^{m} \rightarrow u_{0}$ in $\mathrm{H}_{\pi, \alpha}^{1}(B)$ as $m \rightarrow \infty$ be such that

$$
\sup _{m \in \mathbb{N}}\left\|u_{0}^{m}\right\|_{\mathrm{H}_{\pi, \alpha}^{1}(B)} \leq c<\infty \text {. }
$$

Then the solution $u^{m}\left(\cdot, u_{0}^{m}\right):[0, \infty) \rightarrow \mathrm{H}_{\pi}^{1}(B)$ of (5.1) with initial datum $u_{0}^{m}$ converges to the solution of limiting equation (1.4) with initial datum $u_{0}$.

Proof. It follows from Remark 5.3 that the minimizing movements for $E_{\alpha}^{m}$ and $E_{\alpha}$ coincide with the solutions $T_{\alpha}^{m}(t) u_{0}^{m}$ and $T_{\alpha}(t) u_{0}$ given by the analytic semigroups $T_{\alpha}^{m}$ and $T_{\alpha}$ generated by the operators $A_{\alpha}^{m}=\nabla \cdot\left(\left[\frac{1}{m}+\alpha\right] \nabla \cdot\right)$ and $A_{\alpha}$ on $\mathrm{L}_{\pi}^{2}(B)$, respectively.

Now equi-coercivity follows from

$$
E_{\alpha}^{m}(u) \geq E_{\alpha}(u), u \in \mathrm{L}_{\pi}^{2}(B)
$$

and the coercivity on $E_{\alpha}$ on $\mathrm{H}_{\pi, \alpha}^{1}(B)$. As for weak lower semicontinuity of $E_{\alpha}^{m}$, take a sequence $\mathrm{H}_{\pi}^{1}(B) \ni u_{k} \rightarrow u$ in $\mathrm{H}_{\pi, \alpha}^{1}(B)$. It is easily verified that, for any fixed $\varepsilon>0$,

$$
u_{k} \rightarrow u \text { in } \mathrm{H}_{\pi}^{1}\left(T_{\varepsilon}(\Gamma)^{\mathrm{c}}\right) \text { as } k \rightarrow \infty
$$

where, as before, $T_{\varepsilon}(\Gamma)$ is the tubular neighborhood of $\Gamma$ of "thickness" $\varepsilon>0$. It follows that, for any fixed $\varepsilon>0$,

$$
\begin{aligned}
\int_{T_{\varepsilon}(\Gamma)^{c}}\left[\frac{1}{m}+\alpha\right]|\nabla u|^{2} d x & \leq \liminf _{k \rightarrow \infty} \int_{T_{\varepsilon}(\Gamma)^{\mathrm{c}}}\left[\frac{1}{m}+\alpha\right]\left|\nabla u_{k}\right|^{2} d x \\
& \leq \liminf _{k \rightarrow \infty} \int_{B}\left[\frac{1}{m}+\alpha\right]\left|\nabla u_{k}\right|^{2} d x .
\end{aligned}
$$

Thus, if $u \in \mathrm{H}_{\pi}^{1}(B)$, then

$$
\int_{B}\left[\frac{1}{m}+\alpha\right]|\nabla u|^{2} d x=\lim _{\varepsilon \rightarrow 0+} \int_{T_{\varepsilon}(\Gamma)^{\mathrm{c}}}\left[\frac{1}{m}+\alpha\right]|\nabla u|^{2} d x \leq \liminf _{k \rightarrow \infty} \int_{B}\left[\frac{1}{m}+\alpha\right]\left|\nabla u_{k}\right|^{2} d x,
$$

whereas, if $u \in \mathrm{H}_{\pi, \alpha}^{1}(B) \backslash \mathrm{H}_{\pi}^{1}(B)$, one has that

$$
\liminf _{k \rightarrow \infty} \int_{B}\left[\frac{1}{m}+\alpha\right]\left|\nabla u_{k}\right|^{2} d x \geq \int_{T_{\varepsilon}(\Gamma)^{\mathrm{c}}}\left[\frac{1}{m}+\alpha\right]|\nabla u|^{2} d x \rightarrow \infty \text { as } \varepsilon \rightarrow 0
$$


Remark 5.6. Notice that the existence of approximating sequences for intial data such as those needed for Theorem 5.5 follows from the construction of recovery sequences performed in the proof of Proposition 5.1.

\section{Appendix}

It remains to prove that (1.3) and (1.5) are valid. It is well-known that

$$
\mathcal{F}^{-1}\left(\frac{1}{|\xi|^{\varepsilon}}\right)=\frac{c_{\varepsilon}}{|x|^{n-\epsilon}}
$$

on $\mathbb{R}^{n}$ for $n=1,2$. Using this and classical arguments based on the Poisson summation formula it can be inferred that, for the discrete Fourier transform of periodic functions

$$
\mathcal{F}^{-1}\left(\frac{1}{|k|^{\varepsilon}}\right)=\frac{c_{\varepsilon}}{|x|^{n-\epsilon}}+h_{\varepsilon}(x)
$$

for a $\mathrm{C}^{\infty}$-function $h_{\varepsilon}$. Indeed we have the following kernel characterizations.

Lemma 6.1. Let $\epsilon \in(0,1)$ and assume that the fractional derivative be given by

$$
|\nabla|^{-\varepsilon}|\nabla u|=\mathcal{F}^{-1} \operatorname{diag}\left\{\frac{1}{|k|^{\epsilon}}\right\} \mathcal{F}(|\nabla u|)=N_{\varepsilon}(|\nabla u|),
$$

where $\nabla$ is taken to be $\partial$ when $n=1$. Then, for $n=1,2$,

$$
|\nabla|^{-\varepsilon}|\nabla u|=\int_{B} G_{\varepsilon}^{n}(x-\tilde{x})|\nabla u|(\tilde{x}) d \tilde{x}
$$

for a periodic function $G_{\epsilon}^{n}$ satisfying

$$
G_{\epsilon}^{n}(x)=c_{\epsilon} \frac{1}{|x|^{2-\epsilon}}+h_{\epsilon}^{n}(x), x \in B^{n},
$$

and a function $h_{\epsilon}^{n} \in \mathrm{C}^{\infty}$.

Proof. By definition, one has that

$$
\widehat{G}_{\epsilon}^{n}(k)=\frac{1}{|k|^{\epsilon}}, k \in \mathbb{Z}_{*}^{2}:=\mathbb{Z}^{n} \backslash\{0\} .
$$

This means that

$$
G_{\epsilon}^{n}(x)=\sum_{k \in \mathbb{Z}_{*}^{n}} \frac{1}{|k|^{\epsilon}} e^{\pi i k \cdot x}=\sum_{k \in \mathbb{Z}_{*}^{n}} \frac{\eta(k)}{|k| \epsilon} e^{\pi i k \cdot x},
$$

where $\eta \in \mathrm{C}^{\infty}\left(\mathbb{R}^{n}\right)$ is a cut-off function with

$$
\eta(x)= \begin{cases}0, & |x| \leq 1 / 4 \\ 1, & |x| \geq 1 / 2 .\end{cases}
$$

Notice that Poisson summation formula yields

$$
G_{\epsilon}^{n}(x)=\sum_{k \in \mathbb{Z}_{*}^{n}} \frac{\eta(k)}{|k|^{\epsilon}} e^{\pi i k \cdot x}=g_{\epsilon}^{n}(x)+\sum_{k \in \mathbb{Z}_{*}^{n}} g_{\epsilon}^{n}(x+k), x \in B^{n},
$$

where $g_{\epsilon}^{n}=\mathcal{F}\left(\eta|\cdot|^{-\epsilon}\right)$ is rapidly decreasing (faster than the reciprocal of any polynomial) as the Fourier transform of a smooth function, and satisfies

$$
g_{\epsilon}^{n}=c_{\epsilon}|\cdot|^{\epsilon-1}+\mathcal{F}\left([\eta-1]|\cdot|^{-\epsilon}\right), x \in \mathbb{R},
$$


where the second addend is a smooth function as the Fourier transform of a compactly supported function. Combining everything together yields the claimed decomposition with

$$
h_{\epsilon}^{n}=\mathcal{F}\left([\eta-1]|\cdot|^{-\epsilon}\right)+\sum_{k \in \mathbb{Z}^{*}} g_{\epsilon}^{n}(\cdot+k) .
$$

The following lemma gives a proof of (1.5).

Lemma 6.2. If $n=1$, set $u_{0}=\chi_{[-1 / 2,1 / 2]}$ (or the characteristic function of any interval) and, if $n=2$, let $u_{0}=\chi_{\Omega}$ for a domain $\Omega \subset B$ with smooth boundary $\Gamma$ (or a finite combination of such characteristic functions of non-intersecting domains). Then, for $n=1,2$, with the same interpretations as in the previous lemma, one has that

$$
|\nabla|^{-\varepsilon}\left|\nabla u_{0}\right|(x) \sim d(x, \Gamma)^{\varepsilon-1} \text { for } d(x, \Gamma) \sim 0 .
$$

Proof. Using the kernel representation given in Lemma 6.1 and the fact that $\partial u_{0}=\delta_{-1 / 2}-\delta_{1 / 2}$ yields that

$$
\left(G_{\epsilon}^{1} *_{\pi}\left|\partial u_{0}\right|\right)(x)=c_{\epsilon}\left[\frac{1}{|x+1 / 2|^{1-\epsilon}}+\frac{1}{|x-1 / 2|^{1-\epsilon}}\right]+\text { smooth term, } x \in(-1,1),
$$

and the claims follow. When $n=2$, it is easily seen that $\nabla \chi_{\Omega}=\nu_{\Gamma} \delta_{\Gamma}$ for

$$
\left\langle\nu_{\Gamma} \delta_{\Gamma}, \varphi\right\rangle=\int_{\Gamma} \nu_{\Gamma}(x) \cdot \varphi(x) d \sigma_{\Gamma}(x)
$$

and where $\nu_{\Gamma} \delta_{\Gamma}$ can be interpreted as a vector measure. Then its total variation measure $\left|\nu_{\Gamma} \delta_{\Gamma}\right|$ is simply given by $\delta_{\Gamma}$. It follows that

$$
N_{\epsilon}\left(\left|\nabla u_{0}\right|\right)=\int_{B} G_{\varepsilon}^{2}(x-\tilde{x})\left|\nabla u_{0}\right|(\tilde{x}) d \tilde{x}=c_{\varepsilon} \int_{\Gamma} \frac{1}{|x-\tilde{y}|^{2-\varepsilon}} d \sigma_{\Gamma}(\tilde{y})+\text { smooth term }
$$

Next fix a point $x$ in the vicinity of $\Gamma$. Denote by $y_{x}$ the point on $\Gamma$ closest to $x$. Exploiting the fact that the curve $\Gamma$ is smooth and compact and has hence bounded curvature, it is seen that

$$
|x-\tilde{y}|^{2}=\left(\left|x-y_{x}\right| \pm\left|\tilde{y}_{x}-\tilde{y}\right|\right)^{2}+\left|y_{x}-\tilde{y}\right|^{2} \sim\left(r \pm c s^{2}\right)^{2}+s^{2} \sim r^{2}+s^{2}
$$

for $\tilde{y}$ in a small fixed ball $\mathbb{B}_{\Gamma}\left(y_{x}, \delta\right)$ uniformly in $x \in T_{\delta}(\Gamma)$ for a (without loss of generality) common $\delta>0$. Here $r=d(x, \Gamma)$ and $s=\left|y_{x}-\tilde{y}_{x}\right|$ where $\tilde{y}_{x}$ is the orthogonal projection of $\tilde{y}$ to the line spanned by $\tau\left(y_{x}\right)$ in the local coordinate system given by $\tau\left(y_{x}\right)$ and $\nu\left(y_{x}\right)$, the unit tangent and outward normal to $\Gamma$ at $y_{x}$, respectively. See figure below. It follows that

$$
N_{\epsilon}\left(\left|\nabla u_{0}\right|\right)(x) \sim \int_{-\delta}^{\delta}\left(s^{2}+r^{2}\right)^{\varepsilon / 2-1} d s \sim r^{\varepsilon-1} \int_{-\infty}^{\infty}\left(1+\sigma^{2}\right)^{\varepsilon / 2-1} d \sigma=c d(x, \Gamma)^{\varepsilon-1},
$$

which yields the claim since $\varepsilon<1$.

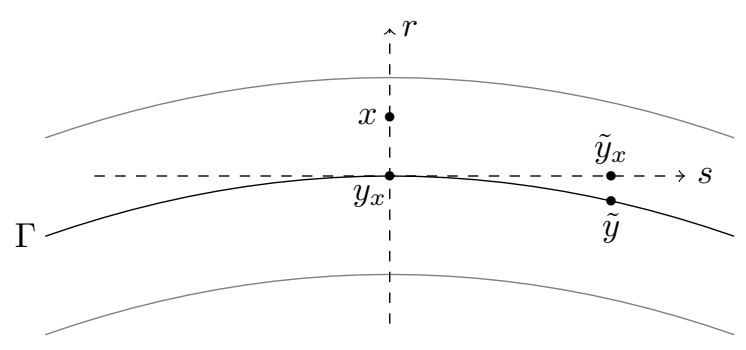




\section{REFERENCES}

[1] H. Amann. Parabolic equations on uniformly regular Riemannian manifolds and degenerate initial boundary value problems, volume Recent Developments of Mathematical Fluid Mechanics of Advances In Mathematical Fluid Mechanics. Birkhäuser Verlag, 2016.

[2] A. Braides. Local Minimization, Variational Evolution and $\Gamma$-Convergence, volume 2094 of Lecture Notes in Mathematics. Springer Verlag, 2014.

[3] G. Fragnelli and J. A. Goldstein G. Ruiz Goldstein. Generators with Interior Degeneracy on Spaces of L ${ }^{2}$-Type. Electron. J. of Differential Equations, 189:1-30, 2012.

[4] J. A. Goldstein. Semigroups of Linear Operators and Applications. Oxford University Press, Oxford, 1985.

[5] P. Guidotti. Numerical Approximation of Generalized Functions: Aliasing, the Gibbs Phenomenon and a Numerical Uncertainty Principle. In Functional Analysis and Evolution Equations, Volume Dedicated to the Memory of Günther Lumer. Birkhäuser, 2007.

[6] P. Guidotti. A family of nonlinear diffusions connecting Perona-Malik to standard diffusion. Discrete and Continuous Dynamical Systems - Series S, 5(3), 2012.

[7] P. Guidotti. Anisotropic Diffusions of Image Processing From Perona-Malik on, 2015.

[8] P. Guidotti and J. Lambers. Two New Nonlinear Nonlocal Diffusions for Noise Reduction. Journal of Mathematical Imaging and Vision, 33(1):25-37, 2009.

[9] P. Guidotti and Y. Shao. Local Well-posedness of a Nonlocal Nonlinear Diffusion Equation of Image Processing. In preparation.

[10] A. Kufner and A. M. Sändig. Some Applications of Weighted Sobolev Spaces. Teubner-Texte zur Mathematik. Springer, Leipzig, 1987.

University of California, Irvine, Department of Mathematics, 340 Rowland Hall, Irvine, CA 926973875 , USA

E-mail address: gpatrick@math.uci.edu 